In Advances in Solid State Physics, Vol. 42, B. Kramer Ed. Springer (2002); ISBN: 3-540-42907-7.

Preprint ref: mpi-pks/0201005

March 12, 2002

\title{
The Role of Contacts in Molecular Electronics ${ }^{\star}$
}

\author{
Gianaurelio Cuniberti ${ }^{1 \star \star}$, Frank Großmann ${ }^{2}$, and Rafael Gutiérrez ${ }^{2}$ \\ 1 Max Planck Institute for the Physics of Complex Systems, \\ D-01187 Dresden, Germany \\ 2 Institute for Theoretical Physics, Technical University of Dresden, \\ D-01062 Dresden, Germany
}

\begin{abstract}
Molecular electronic devices are the upmost destiny of the miniaturization trend of electronic components. Although not yet reproducible on large scale, molecular devices are since recently subject of intense studies both experimentally and theoretically, which agree in pointing out the extreme sensitivity of such devices on the nature and quality of the contacts. This chapter intends to provide a general theoretical framework for modelling electronic transport at the molecular scale by describing the implementation of a hybrid method based on Green function theory and density functional algorithms. In order to show the presence of contact-dependent features in the molecular conductance, we discuss three archetypal molecular devices, which are intended to focus on the importance of the different sub-parts of a molecular two-terminal setup.
\end{abstract}

\section{Introduction}

The incessant development of single molecule techniques is forcing a paradigm shift in the many neighboring branches of nano-sciences. This process does not exclude the modelling and design of electronic devices. Novel fabrication methods that create metallic contacts to a small number of conjugated organic molecules allow the study of the basic transport mechanism of these systems and will provide direction for the potential development of molecularscale electronic systems [1]. The concept is now realized for individual components, but the economic fabrication of complete circuits at the molecular level remains challenging because of the difficulty of connecting molecules to one another. A possible solution to this problem is 'mono-molecular' electronics, in which a single molecule will integrate the elementary functions and interconnections required for computation [2]. Indeed, the primary problems facing the molecular electronics designer are measuring and predicting electron transport. That is due to the fact that molecular electronics is strongly dependent on the quality and nature of the contacts [3]. Ideally, these contacts should be ohmic so that any non-linearity in the conductivity of the wire

\footnotetext{
* This chapter is based on two invited talks (from GC and FG) given at the DPG Spring Meeting (March 2002; Regensburg, Germany)

** Corresponding author: cunibert@mpipks-dresden.mpg.de
} 
can be correctly attributed and studied. They must also be low in resistance to ensure that the properties measured are those of the molecule and not those of the molecule-contact interface. Moreover, the medium surrounding and supporting the molecule must be several orders of magnitude more insulating than the molecule itself because the contact area of the support with the electrical contacts is often much greater than that between the electrical contacts and the molecule [4].

Nevertheless, the contact problem can be turned into a challenge. Even with the intrinsic barrier that the contacts represent, barriers can be strategically used to favor the design of specific devices [5]. However, this requires a more detail account of the atomic structure of the interface. Green function and density functional theories [6] are the typical instruments to characterize transport through single molecules clamped between two metallic contacts. These very same instruments may even be adopted for calculating electromechanical switch behaviors [7] and current-induced forces [8] in molecular structures.

In this chapter, after a brief overview on charge transport on the molecular scale (Sect. 2), we provide, in Sect. 3, a general theoretical hybrid method based on Green function theory and density functional theory (DFT)-based algorithms. In order to show the presence of contact-dependent features in the molecular conductance, we introduce, in Sect. 4, three model molecular devices. The first is a sodium wire (Par. 4.1), where the role of contacts for a molecular bridge emerges clearly. However, the quality of contacts is not the only source of alteration of the molecular conductance. In the Par. 4.2, we show the peculiar effect that carbon nanotube leads might have on a contacted molecule. Finally, in the Par.4.3, a pure carbon device, consisting of two carbon nanotube leads grasping a $\mathrm{C}_{60}$ molecule is studied in a parameter free DFT calculation.

\section{Charge Transport on the Molecular Scale}

In mesoscopic electron transport, many interesting interference related and quantization effects have been found in the past 20 years [9]. Much of the fundamental theory for mesoscopic systems can be taken over to the description of molecular scale conductance calculations. In both realms, a formulation that includes interference effects due to phase coherence as well as geometrical effects is needed. It was originally developed by Landauer [10 for a two-terminal geometry as displayed in Fig. 11. and further extended by Büttiker 11 to the multi-terminal case. The essential idea of the Landauer formulation is to relate the conductance to an elastic scattering problem and, ultimately, to transmission probabilities. The simplest way to derive this relationship is to consider a 'molecular' region connected to two ballistic leads, which are connected to electronic reservoirs at the chemical potentials $\mu_{\mathrm{L}}, \mu_{\mathrm{R}}$, see Fig. 1. It is assumed that electrons entering the reservoirs do 


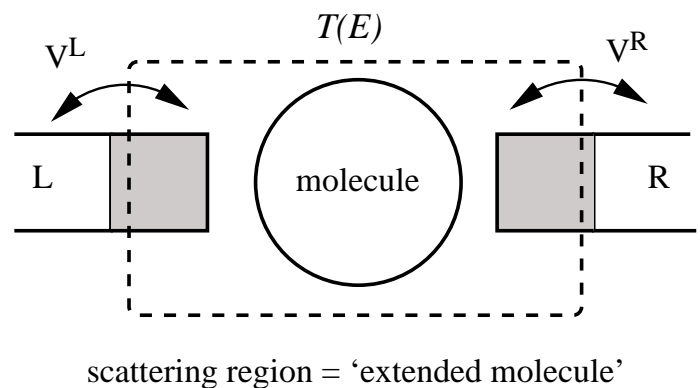

Fig. 1. Schematic representation of a two-terminal device. The scattering region (enclosed in the dashed-line frame) with transmission probability $T(E)$ is connected to semi-infinite left $(\mathrm{L})$ and right $(\mathrm{R})$ leads which end into electronic reservoirs (not shown) at chemical potentials $\mu_{\mathrm{L}}, \mu_{\mathrm{R}}$. By applying a small potential difference electronic transport will occur. The scattering region or molecule may include in general parts of the leads (shaded areas). This is necessary for the description of real systems, where the surface topology may be modified as a result of relaxation or reconstruction processes. This may introduce additional scattering due, e.g., to surface states.

completely lose their phase coherence. As stated in Ref. 12] assuming semiinfinite leads is enough to warrant that no electron exiting the scattering region will reenter it with the same phase, so that an explicit modeling of the reservoirs is not necessary. In equilibrium $\mu_{\mathrm{L}}=\mu_{\mathrm{R}}$, but if an infinitesimal voltage $e V=\mu_{\mathrm{L}}-\mu_{\mathrm{R}}$ is applied a non-equilibrium situation is induced and a current will flow. The scattering region is characterized by the energydependent transmission coefficient $T(E)$. In the zero-temperature, linear response $(\mathrm{eV} \rightarrow 0)$ regime it is found [13] that the proportionality relation,

$$
g=\frac{e^{2}}{\pi \hbar} T\left(E_{\mathrm{F}}\right), \quad T=\sum_{m, n=1}^{N_{\mathrm{F}}}\left|t_{m n}\right|^{2}
$$

holds. $E_{\mathrm{F}}$ is the Fermi energy of the whole system in equilibrium and the transmission amplitudes $t_{m n}$ describe the scattering of one electron from channel $n$ in the left lead to channel $m$ in the right lead. They can be extracted, e.g., from the scattering matrix. The sums run over all $N_{\mathrm{F}}$ open channels at the Fermi level (whose number is assumed to be equal on both sides). Channels (transverse modes) appear due to the finite cross section of the leads which induces quantization of the electronic states perpendicular to the direction of current transport. For the special case of ideal transmission, i. e. $\sum_{n}\left|t_{m n}\right|^{2}=1 \forall m$, the conductance is simply proportional to $N_{\mathrm{F}}$ with the von Klitzing conductance quantum $g_{\mathrm{K}}=e^{2} /(\pi \hbar)$ as the proportionality factor. Thus, $g / g_{\mathrm{K}}$ shows unit steps as a function of $N_{\mathrm{F}}$. This is the wellknown fact of conductance quantization 13 , shown experimentally by van Wees et al. [14] and Wharam et al. 15]. 
In molecular conductance, in the case of strong coupling, it is the electronic structure of the molecule influenced by the leads that determines the transmission properties which in turn play the decisive role for electron transport. The calculation of the coupling to the leads together with the calculation of the electronic structure will be dealt with in the following using DFT-based methodology.

\section{Method}

For a general scattering region where inelastic effects are included, Meir and Wingreen [16] used non-equilibrium Green functions to derive an expression for the current which reduces to Eq. (1) in the elastic case. An advantage of their derivation is that an explicit connection to the Green function $\mathbf{G}$ of the scattering region dressed by the presence of the leads is established. The latter are introduced as self-energy corrections into the bare 'molecular' Green function $\mathbf{G}^{-1}=\mathbf{G}^{\mathrm{M}^{-1}}+\boldsymbol{\Sigma}^{\mathrm{L}}+\boldsymbol{\Sigma}^{\mathrm{R}}$, The result for the transmission probability is then given by 16

$$
T(E)=4 \operatorname{Tr}\left\{\boldsymbol{\Delta}^{\mathrm{L}}(E) \mathbf{G}(E) \boldsymbol{\Delta}^{\mathrm{R}}(E) \mathbf{G}^{\dagger}(E)\right\},
$$

where $\Delta^{\mathrm{L}}, \Delta^{\mathrm{R}}$ are the spectral density describing the coupling of the scattering region to the $\alpha(=\mathrm{L}, \mathrm{R})$-lead given by

$$
\boldsymbol{\Delta}_{\alpha}=\frac{\mathrm{i}}{2}\left(\boldsymbol{\Sigma}_{\alpha}\left(E+\mathrm{i} 0^{+}\right)-\boldsymbol{\Sigma}_{\alpha}^{\dagger}\left(E+\mathrm{i} 0^{+}\right)\right)
$$

and the trace is to be taken over states in the scattering region. The Green function $\mathbf{G}^{\mathrm{M}}$ is in general defined as the inverse operator $\left(E+\mathrm{i}^{+}-\mathbf{H}^{\mathrm{M}}\right)^{-1}$, for some suitable 'molecular' Hamiltonian $\mathbf{H}^{\mathrm{M}}$. Similar expressions have been derived by Fisher and Lee and Todorov, Briggs and Sutton [17. In a seminal paper Szafer and Stone have derived the Landauer result, Eq. (11) from Kubo's linear response theory [18].

As mentioned above, only components of the Green function in the Hilbert subspace associated with the scattering region, which we will denote as 'the molecule' from now on to keep in mind that transport through molecular scale systems is the main issue to be addressed here, are needed. Notice, however, that the molecule may also include some atoms belonging to the leads, see Fig. 1. This will be the case when investigating real systems, where the surface atomic structure of the leads is explicitly taken into account (clean surfaces are usually energetically unstable, so that upon structural relaxation the surface topology may be modified and this will introduce additional scattering). From the full Green function of the open (infinite) system consisting of the leads plus the molecule it is possible to extract $\mathbf{G}$ using projector operator techniques [19]. In order to do so, one partitions the whole system into three components as shown in Fig. 11., where a left electrode, the molecule, and 
a right electrode are depicted. The full associated Hamiltonian matrix (in a suitable basis representation) can then be formally written as

$$
\mathbf{H}=\left(\begin{array}{ccc}
\mathbf{H}^{\mathrm{L}} & \mathbf{V}^{\mathrm{L}, \mathrm{M}} & \mathbf{0} \\
\mathbf{V}^{\mathrm{L}, \mathrm{M}^{\dagger}} & \mathbf{H}^{\mathrm{M}} & \mathbf{V}^{\mathrm{R}, \mathrm{M}} \\
\mathbf{0} & \mathbf{V}^{\mathrm{R}, \mathrm{M}^{\dagger}} & \mathbf{H}^{\mathrm{R}}
\end{array}\right)
$$

The matrices $\mathbf{V}^{\mathrm{L}, \mathrm{M}}, \mathbf{V}^{\mathrm{R}, \mathrm{M}}$ couple atoms belonging to the left(right) leads to the molecule, and it has been assumed that no direct lead-lead coupling exists. Notice that $\mathbf{H}^{\mathrm{L}(\mathrm{R})}$ are infinite dimensional sub-matrices. By means of a operator projecting onto the 'molecular' subspace, we can write the resulting $M$-dimensional matrix equation as:

$$
\left(z \mathbf{S}^{\mathrm{M}}-\mathbf{H}^{\mathrm{M}}-\boldsymbol{\Sigma}^{\mathrm{L}}(z)-\mathbf{\Sigma}^{\mathrm{R}}(z)\right) \mathbf{G}(z)=\mathbf{1}, \quad z=E+\mathrm{i} 0^{+},
$$

where $\mathbf{S}^{\mathrm{M}}$ is the overlap matrix for the general case of a non-orthogonal basis set. The energy-dependent self-energies $\boldsymbol{\Sigma}^{\mathrm{L}}, \boldsymbol{\Sigma}^{\mathrm{R}}$ include the coupling to the leads as well as information on the electronic structure of the leads. For the $\alpha$-lead, they are given by

$$
\boldsymbol{\Sigma}^{\alpha}(z)=\left(z \mathbf{S}^{\alpha, \mathrm{M}}-\mathbf{V}^{\alpha, \mathrm{M}}\right)^{\dagger} \mathcal{G}^{\alpha}(z)\left(z \mathbf{S}^{\alpha, \mathrm{M}}-\mathbf{V}^{\alpha, \mathrm{M}}\right) .
$$

The matrix $\mathbf{S}^{\alpha}$ is the overlap matrix element between molecule atoms and the $\alpha$-lead atoms and $\mathcal{G}^{\alpha}(z)$ is the $\alpha$-lead surface Green functions. Since the coupling matrices are in general short-ranged they will eliminate all contributions coming from atoms other than those closest to the molecule. Hence, only surface Green functions are usually needed.

We would like to stress that Eqs. (11) and (2) are only valid in the case that inelastic processes in the scattering region can be completely neglected. Otherwise no simple relationship between conductance and transmission can be obtained. A typical example where electron-electron interactions are decisive are quantum dots. There, the scattering region is weakly coupled to the leads so that the coupling-induced level broadening will be much smaller than the charging energy. Hence, electron interaction effects leading, e.g., to Coulomb blockade phenomena should be included in the description of quantum transport 20].

At this point we are led to the issue of characterizing the electronic structure of the molecule as well as of the leads. If we only focus on the essential physics, some kind of model Hamiltonians can be used [21. However, if real situations are addressed where the knowledge of the detailed electronic structure is important, the use of more realistic computational schemes is unavoidable. From the point of view of electronic structure calculations three classes of approaches have been implemented for quantum transport calculations:

(i) Semiempirical or empirical tight-binding (TB) schemes, e.g. (extended) Hückel Hamiltonians, where the matrix elements are fitted to experiments or to first-principle calculations [22]. 


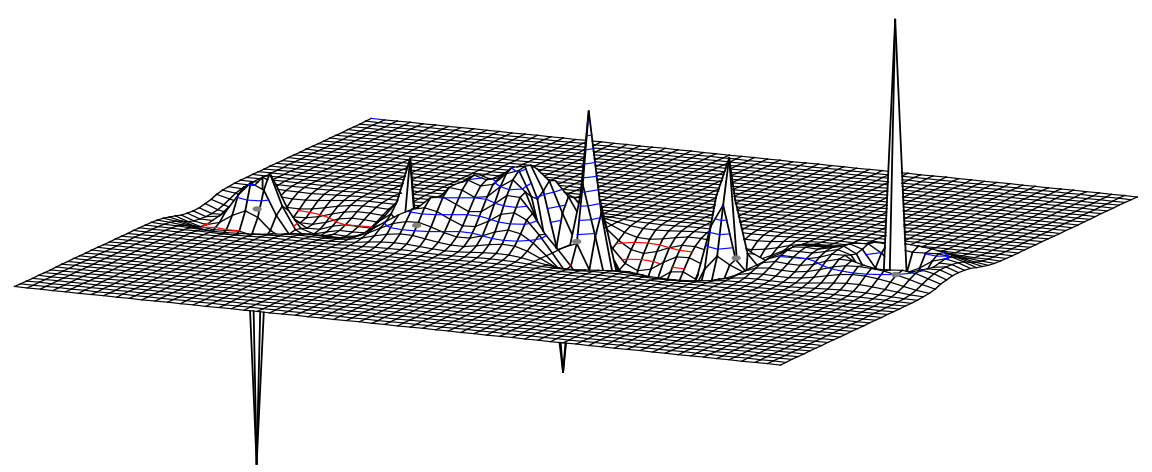

Fig. 2. A five sodium atom isolated wire. The HOMO density is plotted in arbitrary units.

(ii) First-principles or ab initio approaches like Hartree-Fock and (DFT) [23].

(iii) Schemes which combine some elements of points (i) and (ii) in firstprinciples parametrized tight-binding Hamiltonians as it is the case for TB-DFT [7,24,25,26] methods.

Concerning the last class mentioned above, a computational scheme has been developed in Ref. 25] which combines a DFT-parametrized TB approach with the Landauer formalism to study the electronic transport properties of sodium atomic chains [24, small sodium clusters 25, carbon-based molecular junctions [7] as well as to simulate Scanning-Tunneling-Spectroscopy experiments on organic molecules [26]. The TB-DFT scheme relies on a representation of the electronic eigenstates of the system within a non-orthogonal localized basis set, usually taken as a valence basis. The many-body Hamiltonian is then approximately represented by a two-center tight-binding Hamiltonian. The matrix elements, however, are calculated numerically, avoiding the introduction of empirical parameters as in conventional TB approaches. We will now discuss some of the applications of this combined scheme.

\section{Applications to Molecular Devices}

\subsection{Focusing on the Bridge Molecule: Sodium Wires}

In this section, we review the numerical results for the resistance $\mathcal{R}=1 / g$ of sodium atomic wires as a function of the electrode-wire separation $d$ and of the wire length 24]. The bond length in the wires was fixed at $6.00 a_{\mathrm{B}}$, which approximately corresponds to the equilibrium distance of a Na-dimer $\left(d_{\mathrm{eq}}=5.67 a_{\mathrm{B}}\right)$. For wires with more than four atoms dimerization of the wire is expected due to a Peierls transition. Such effects will not be considered here. 


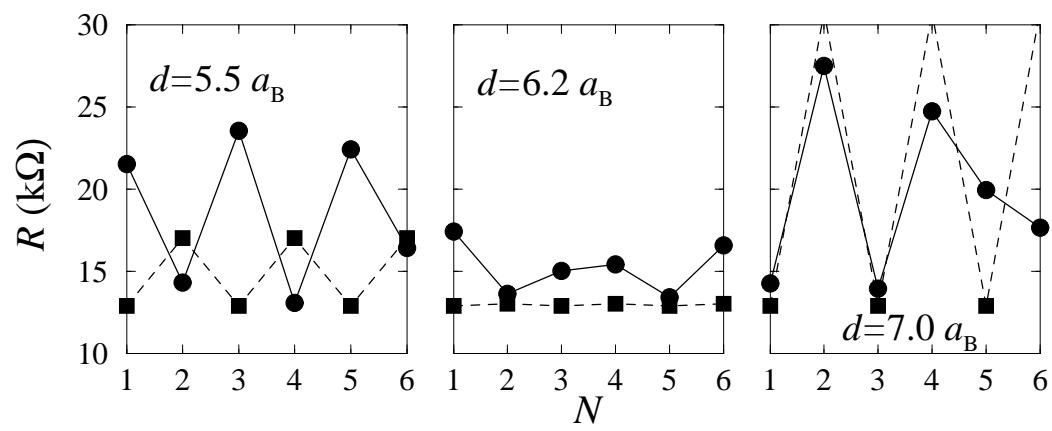

Fig. 3. Dependence of the resistance on the length of the atomic sodium wire for different electrode-wire separations. Dashed lines (connecting the squares) correspond to a resistance calculated with only the 3s-valence orbitals in the wire.

In Fig. 3 ., the dependence of the resistance $\mathcal{R}=1 / g$ on the number of atoms in the chain is displayed for three different values of $d$. The result of Lang, who stated that $R_{N=1}>R_{N=2}$ [27], is only found, in our approach, in the case of strong coupling between the chain and the electrode. Concerning the coupling strength, there exists a critical value $d_{\text {crit }}$ where both the single atom and the dimer have approximately the same resistance. This behaviour can be understood by inspecting the transmission spectrum, as shown in Fig. 1 . The value, the linear resistance of the wire acquires, depends sensitively on the position of the Fermi level $E_{\mathrm{F}}$ with respect to the modified eigenenergies of the wire. In order to distinguish between the bare eigenenergies we have displayed the free wire density of states (DOS) together with the corresponding $T(E)$ for two different values of the electrode-wire separation. Intuitively one would expect that $E_{\mathrm{F}}$ lies in the HOMO-LUMO gap for a Na-dimer (the HOMO is twice occupied) and would almost touch the singly occupied HOMO in the one atom case. This picture is, however, only exact in the case of a weak coupling to the electrodes, where the position of the eigenvalues of the wire remains approximately the same as for an isolated wire and the broadening induced by the coupling is smaller than the energy spacing between the eigenvalues. For $d=6.2 a_{\mathrm{B}}$, however, the eigenstates of $\mathrm{Na}_{1}$ and $\mathrm{Na}_{2}$ are strongly broadened and shifted by the coupling to the leads. The HOMO and LUMO (3fold degenerate) of the single atom cannot be clearly resolved any more but evolve into a rather broad single peak. Especially at $E_{\mathrm{F}}$ the transmission for a single atom becomes smaller than for the dimer. With increasing distance the coupling to the electrodes is reduced and thus the renormalization and broadening of the eigenstates become weaker. At $d=7.0 a_{\mathrm{B}}$, the HOMO and LUMO of the dimer are already 'resolved' and the transmission $T\left(E_{\mathrm{F}}\right)$ within the gap is reduced.

In this section, we have introduced as possible bridge molecule a sodium wire. There the simplest assumption has been made for the lead self-energy 
entering in the calculation, a semi-infinite linear chain with a semi-elliptical spectral density $\Delta$, obtained by Newns [28]. What are the effects which might arise from using nanoelectrodes such as carbon nanotubes?

\subsection{Focusing on the Leads: Carbon Nanotube Leads}

Carbon nanotube (CNT) conductors have been in the focus of intense experimental and theoretical activity as another promising direction for building blocks of molecular-scale circuits [29,30]. Carbon nanotubes exhibit a wealth of properties depending on their diameter, on the orientation of graphene roll up, and on their topology, namely whether they consist of a single cylindrical surface (single-wall) or many surfaces (multi-wall) [31, 32, Carbon nanotubes have been recently used as wiring elements [30, as active devices 30,33, and, attached to scanning tunneling microscope (STM) tips, for enhancing their resolution 34. With a similar arrangement the fine structure of a twinned DNA molecule has been observed [35]. However, CNT-STM images seem to strongly depend on the tip shape and nature of contact with the imaging substrate 36. If carbon nanotubes are attached to other materials to build elements of molecular circuits, the characterization of contacts 37] becomes again a fundamental issue. This problem arises also when a carbon nanotube is attached to another molecular wire, a single molecule or a molecular cluster. In this section we present analytic results for the transmission through a CNT-molecule-CNT system. In particular we analytically derive the spectral density of a single wall armchair carbon nanotubes, needed for calculating the transmission. A possible configuration is depicted in Fig. f. Here a $N$ atom molecule has been adopted as bridge molecule. However, the results we obtain are valid for any bridge sub conditio that the CNTs are contacting the molecular complex only via two single atomic contacts (labelled here as 1
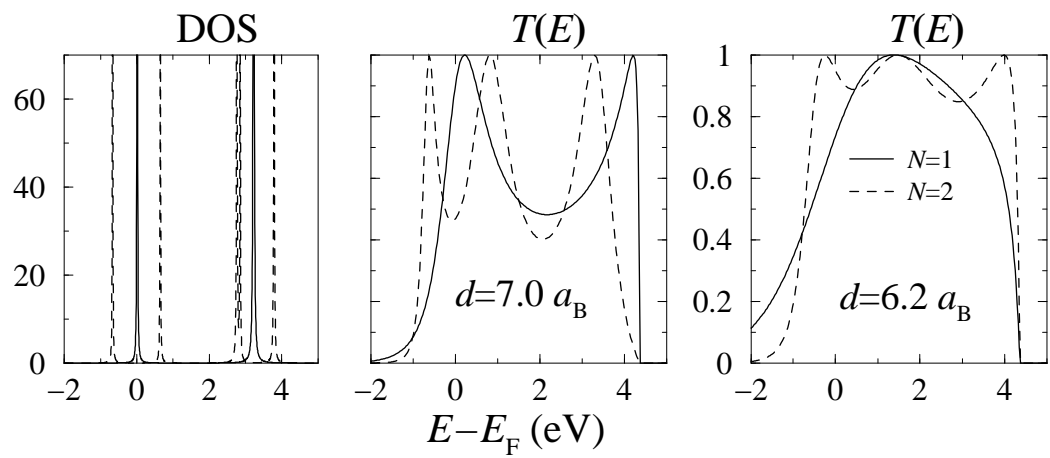

Fig. 4. The transmission coefficient as a function of energy for one and two atoms between the electrodes for two different electrode-wire separations. The left panel shows the DOS of the isolated wires. Only the low-energy part of the spectra is shown. 



Fig. 5. Schematic representation of the $N$ atom molecule-carbon nanotube hybrid with single (bottom) and multiple (top) contacts. on-site energies $\varepsilon^{\alpha=\mathrm{L}, \mathrm{R}}$ are chosen to be zero.

and $N$ ). For the system under investigation, where only the two atoms of the molecule are coupled to the leads, the formula for the transmission simplifies to 38,39

$$
T(E)=4 \Delta^{\mathrm{L}}(E) \Delta^{\mathrm{R}}(E)\left|G_{1 N}^{\mathrm{M}}(E)\right|^{2} / \operatorname{det}(\mathbf{Q}),
$$

where the spectral densities $\Delta^{\mathrm{L}}$ and $\Delta^{\mathrm{R}}$ are the only non-zero elements $\left(\Delta^{\mathrm{L}}\right)_{11}$ and $\left(\Delta^{\mathrm{R}}\right)_{N N}$, respectively, of the matrices $\Delta$. The matrix element $\Delta^{\mathrm{L}(\mathrm{R})}$ is the spectral density of the left (right) lead. It is related to the semiinfinite lead Green function matrix $\mathcal{G}^{\mathrm{L}(\mathrm{R})}$. It is minus the imaginary part of the lead self-energies (per spin),

$$
\Sigma^{\alpha}=\Lambda^{\alpha}-\mathrm{i} \Delta^{\alpha}=\sum_{m_{\alpha}, m_{\alpha}^{\prime}} \Gamma_{m_{\alpha}} \Gamma_{m_{\alpha}^{\prime}}^{*} \mathcal{G}_{m_{\alpha} m_{\alpha}^{\prime}}^{\alpha},
$$

with $\alpha=\mathrm{L}$, R. Owing to the causality of the self-energy, its real part $\Lambda$ can be entirely derived from the knowledge of $\Delta$ via a Hilbert transform. Finally the determinant of $\mathbf{Q}=\mathbf{1}-\mathbf{\Sigma} \mathbf{G}^{\mathrm{M}}$ has to be calculated.

The rhs of Eq. (5) coincides with formulas used to describe electron transfer in molecular systems [21, 40]. The above relationship between the Landauer scattering matrix formalism on the one side and transfer Hamiltonian approaches on the other side has been worked out in the recent past [41] showing, de facto, their equivalence. This enables us to make use of the formulas from a Bardeen-type picture in terms of spectral densities, which is often convenient for an understanding and analysis of the results obtained.

In calculating the spectral function, we make use of the assumption of identical left and right leads and drop the self-energy indices in Eq. (7). Since a $\pi$ orbital representation was found to give good agreement with experiments (even quantitatively) 31], the Hamiltonian at hand can be assumed discrete. We can write the lattice Green function $G=\left(E+\mathrm{i} 0^{+}-\mathbf{H}\right)^{-1}$ in matrix form by rearranging the two dimensional $n$ lattice coordinate with honeycomb underlying structure in the tight-binding Hamiltonian representation. The boundary conditions are imposed on two cuts parallel to a lattice bond so that the surface of a semi-infinite CNT contains $2 \ell$ atoms for a so-called 
$(\ell, \ell)$ armchair CNT. We assume the $x$ direction to be parallel to the tubes (and to the transport direction) and $y$ to be the finite transverse coordinate (see Fig. 5.). The latter is curvilinear with $n_{y}$ spanning the $2 \ell$ sites with periodic boundary conditions.

The lattice representation of the lead Green function is needed in the calculation of the self-energy contribution. It can generally be written by projecting the Green operator onto the localized state basis, $\psi_{k_{x}, k_{y}}\left(n_{x}=\right.$ border, $\left.n_{y}\right)=$ $\chi_{k_{x}} \phi_{k_{y}}\left(n_{y}\right)$, of the semi-infinite lead:

$$
\begin{aligned}
\mathcal{G}_{n_{y} n_{y}^{\prime}}(E) & =\left\langle n_{y}\left|\left(E+\mathrm{i} 0^{+}-\mathbf{H}\right)^{-1}\right| n_{y}^{\prime}\right\rangle \\
& =\sum_{k_{x}, k_{y}} \frac{\chi_{k_{x}} \phi_{k_{y}}\left(n_{y}\right) \chi_{k_{x}}^{*} \phi_{k_{y}}^{*}\left(n_{y}^{\prime}\right)}{E+\mathrm{i} 0^{+}-E_{k_{x}, k_{y}}} .
\end{aligned}
$$

The eigenvalues of the tight-binding Hamiltonian

$$
E_{ \pm}\left(k_{x}^{j}, j\right)=\varepsilon \pm \gamma \sqrt{1+4 \cos \left(\frac{j \pi}{\ell}\right) \cos \left(\frac{k_{x}^{j} a}{2}\right)+4 \cos ^{2}\left(\frac{k_{x}^{j} a}{2}\right)},
$$

are obtained in a basis set given by symmetric (+) and antisymmetric (-) site configurations of the graphene bipartite lattice, corresponding to $\pi$ and $\pi^{*}$ orbitals, respectively 42]. The longitudinal momentum is restricted to the Brillouin zone, $-\pi<k_{x}^{j} a<\pi$, and the transverse wave number $1 \leq j \leq 2 \ell$ labels $4 \ell$ bands, as many as the number of atoms in the unit cell of a $(\ell, \ell)$ CNT. The two bands corresponding to $j=\ell$ are singly degenerate. They are responsible for the metallic character of armchair carbon nanotubes (these two bands cross at the Fermi level $E=\varepsilon$ for $\left.k_{x}^{\ell} a= \pm 2 \pi / 3\right)$. Also the two outermost bands corresponding to $j=2 \ell$ are singly degenerate, while the other remaining $(4 \ell-4)$ bands are collected in $(2 \ell-2)$ doubly-degenerate dispersion curves.

The single-particle Green function in a lattice representation for two sites belonging to the same sub-lattice can be written as

$$
\begin{aligned}
\mathcal{G}_{n_{y} n_{y}^{\prime}}(E) & =\frac{a}{2 \pi \ell} \sum_{j, \beta} \int_{-\pi / a}^{\pi / a} \mathrm{~d} k_{x}^{j} \frac{\sin ^{2}\left(k_{x}^{j} a\right) \varphi_{j}\left(n_{y}\right) \varphi_{j}^{*}\left(n_{y}^{\prime}\right),}{E+\mathrm{i} 0^{+}-E_{\beta}\left(k_{x}^{j}, j\right)} \\
& =\frac{1}{2 \ell} \sum_{j=1}^{2 \ell} \varphi_{j}\left(n_{y}\right) \tilde{G}^{j}(E) \varphi_{j}^{*}\left(n_{y}^{\prime}\right),
\end{aligned}
$$

where $\varphi_{j}\left(n_{y}\right)=\exp \left(\mathrm{i} k_{y}^{j} n_{y} a\right)$, with $k_{y}^{j} a=\pi j / \ell$, and $1 \leq j \leq 2 \ell$. Note that in Eq. (10), $n_{y}$ and $n_{y}^{\prime}$ should be either even or odd (that is they should belong to the same sublattice). The semi-infinite longitudinal Green function is given by

$$
\tilde{G}^{j}(E)=\frac{a}{8 \pi} \sum_{\beta= \pm} \int_{-\pi / a}^{\pi / a} \mathrm{~d} k_{x}^{j} \frac{\sin ^{2}\left(k_{x}^{j} a / 2\right)}{E+\mathrm{i} 0^{+}-E_{\beta}\left(k_{x}^{j}, j\right)} .
$$



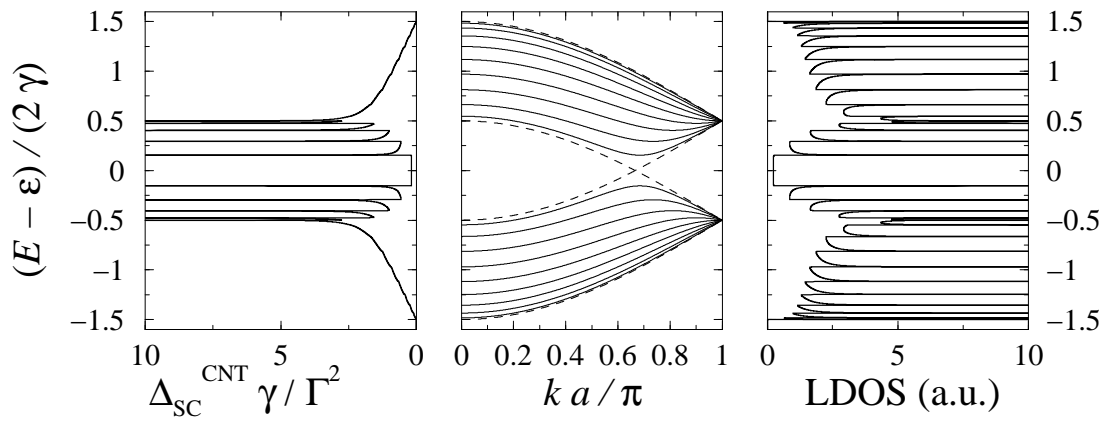

Fig. 6. Left panel: the normalized spectral density for a semi-infinite $(\ell, \ell)$ CNT lead in the SC configuration; it corresponds to the LDOS at any atom site at the cut of the CNT lead. For comparison the dispersion relation and the LDOS of an infinite $(\ell, \ell)$ CNT are shown in the middle and right panel respectively. Solid lines in the dispersion relation panel indicate doubly degenerate bands, dashed lines singly degenerate bands. Here $\ell=10$, and on-site energies and hopping terms refer to $\alpha=\mathrm{L}$, R-leads.

The integral can be worked out analytically by extending $k_{x}^{j}$ to the complex plane and adding cross-cancelling paths (parallel to the imaginary axis) along the semi-infinite rectangle in the half plane $\operatorname{Im} k_{x}^{j}>0$ and based on the interval between $-\pi / a$ and $\pi / a$. The closing path parallel to the real axis gives a real contribution linear in energy. This generalizes the approach by Ferreira et al. [43], recently adopted for obtaining an analytical expression for the diagonal Green function of infinite achiral tubes, to the case of semiinfinite CNTs. The determination of the poles inside the integration contour, given by

$$
-2 \cos \left(\frac{q_{\beta}^{j} a}{2}\right)=\cos \left(\frac{j \pi}{\ell}\right)+\beta \sqrt{\left(\frac{E-\varepsilon}{2 \gamma}\right)^{2}-\sin ^{2}\left(\frac{j \pi}{\ell}\right)},
$$

allows for the calculation of the residues and thus of the surface Green function. One finds

$$
\tilde{G}^{j}(E)=\frac{1}{2 \gamma} \frac{E-\varepsilon}{2 \gamma}\left(1+\mathrm{i} \frac{\sin \left(\frac{q_{\beta_{*}}^{j} a}{2}\right)}{\sqrt{\left(\frac{E-\varepsilon}{2 \gamma}\right)^{2}-\sin ^{2}\left(\frac{j \pi}{\ell}\right)}}\right),
$$

where the choice of the contributing pole through the branch parameter $\beta_{*}=$ $\operatorname{sign}(E-\varepsilon)$ has to be taken into account. The LDOS, obtained from the imaginary part of the surface Green function after Eq. (11) is plugged into Eq. (10), is shown in Fig. 6. It clearly differs from the LDOS of an infinite $\mathrm{CNT}$ as depicted for comparison in the right panel. As for the case of the 
SLT the pinning of the longitudinal wave function at the surface of the semiinfinite systems cancels all border zone anomalies when $q_{ \pm}^{j} a$ matches multiples of $2 \pi$. In infinite SLTs these states are the only resonant states (van Hove singularities) so that the surface LDOS of a semi-infinite SLT never diverges. On the contrary, in CNTs there are states with zero group velocity outside the border zone which are responsible for the singularities of the spectral density of semi-infinite CNTs (left panel of Fig. 6.). The self-energy for a CNT lead is more complicate than the one for a SLT owing to the missing equivalence of the sites belonging to the two different sub-lattices. However, since the longitudinal part of the Green function, Eq. (11), is the same for all diagonal and off-diagonal terms of the surface Green function, the self-energy can still be cast into the form

$$
\Sigma=\frac{1}{2 \ell} \sum_{j=1}^{2 \ell} \tilde{G}^{j}(E) \eta_{j / \ell}[\Gamma] .
$$

However, for the calculation of

$$
\eta_{j / \ell}[\Gamma]=\left|\sum_{m=1}^{2 \ell} \Gamma_{m} \varphi_{j}(m)\right|^{2},
$$

one has to specify the sub-lattice components of the transverse wave function and whether they belong to a bonding or anti-bonding molecular state. Again the distribution of the $\Gamma_{m}$ contacts is needed in oder to calculate the weight $\eta$ and thus the self-energy. Eq. (12) simplifies considerably in the SC case: $\eta=\Gamma^{2}$. Since $\eta$ is uniform in $j$, the self-energy is simply proportional to the diagonal semi-infinite Green function and, as a consequence, the spectral density is proportional to the local density of states (Fig. 6.). The MC case $\left(\Gamma_{m}=\Gamma_{\text {eff }} / \sqrt{2 \ell}\right)$ is also easily tractable leading to a sum rule over the possible conducting channels. However, a direct proof is provided by the intuitive consideration that only the $\pi$-bonding state can contribute to the MC spectral density (all the other states have a non-constant spatial modulation provided, e.g., in Ref. 44). Following our notation, the $\pi$-bonding state corresponds to $j=\ell$. The two different lead lattice structures carry the same physical information only in the MC limit case [38].

\subsection{Focusing on the 'Molecule Plus Lead' Complex: a Pure Carbon Device}

In this section we focus on the combination of CNT-leads with a realistic molecular cluster acting as the central molecule. Especially interesting is the case of a monovalent carbon cluster which makes the system an "all-carbon" device. Therefore, we studied a single $\mathrm{C}_{60}$ molecule bridging two single-wall metallic $(5,5)$ carbon nanotubes (CNT). The CNT were taken symmetric with respect to the plane through the center of mass of $\mathrm{C}_{60}$ and perpendicular to the CNT cylinder axes (see left panel of Fig. 7.). 




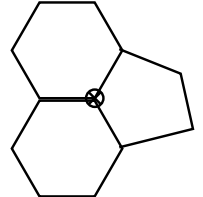

(2)

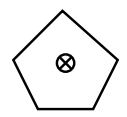

(3)

Fig. 7. Geometric configuration of the carbon molecular junction. $\mathrm{A}_{60}$ molecule bridges two $(5,5)$ CNTs. The right panel represents schematically the different orientations of $\mathrm{C}_{60}$ with respect to the surface cross-sections of the nanotubes (e.g. the left panel geometry corresponds to orientation (1)). The nanotube symmetry axis is depicted by a cross inside a circle.

The central aim of [7] was to exploit the sensitivity of electron transport to the topology of the molecule/electrode interface in the proposed system. In this pure carbon system, charge transfer effects will be negligible. The Fermi level of the whole system will therefore lie within the HOMO-LUMO gap of the isolated $\mathrm{C}_{60}$. Therefore, the electronic transport will be mainly mediated by the overlap of the tails of the molecular resonances within the HOMO-LUMO gap of $\mathrm{C}_{60}$.

The key problem we addressed was how severely orientational effects do influence the electronic transport. To this end several possible orientations of the $\mathrm{C}_{60}$ (depicted by the polygon(s) facing the tube symmetry axis in the

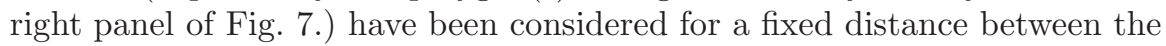
molecule and the tubes. For the sake of comparison, structurally unrelaxed and relaxed molecular junctions were considered. The basic results are displayed in Fig. 8., for both relaxed and unrelaxed structures. Surprisingly, at fixed distance, just an atomic scale rotation of the highly symmetric $\mathrm{C}_{60}$ molecule induces a large variation of the transmission at the Fermi energy by several orders of magnitude. This can be seen in Fig. 8.(right panel) for three different orientations with maximum, minimum and one intermediate value of $T\left(E_{\mathrm{F}}\right)$. As can be seen in Fig. 8. (left panel), neglecting relaxation decisively influences the transmission properties of the molecular junction. This shows up as a different and less smooth behaviour of the transmission. The qualitative difference is related to the presence of dangling bond states on the CNT surfaces. Such states usually lie within a gap (a similar situation as that found, e.g., in semiconductor surfaces), in this case the HOMOLUMO gap of the isolated molecule. They lead to the oscillatory behaviour in the transmission for unrelaxed junctions. Upon relaxation these states are partly saturated or they rehybridize, moving away from the middle of the gap. However, some of them may still lie just above the HOMO or just below the LUMO of $\mathrm{C}_{60}$, giving some contribution to the transmission within the gap. 




Fig. 8. Transmission results for both unrelaxed (left panel) and relaxed (right panel) configurations. The tube-tube distance $d$ is fixed at $0.93 \mathrm{~nm}$. Numbers indicate different molecular orientations as depicted in the right panel of Fig. 7 .

The results for the relaxed structures reveal that, at the Fermi energy, the pentagon configuration (3) has a transmission lower by about three orders of magnitude than configuration (2). This fact could possibly be exploited in an electronic switching device on the nanoscale, as manipulation of fullerenes by using STM or atomic force microscope tips is becoming a standard technique in the field 445].

\section{Discussion and Conclusions}

Summing up our results, we can conclude that modelling transport at the molecular scale cannot avoid to go through an accurate structure calculation of the smallest element in the device, namely, the molecular bridge and part of the attached leads. Ab initio methods, although approximated on the DFTLDA level description, provide thus a fundamental input to be integrated in standard quantum transport techniques. The hybrid quantum-transport-abinitio method, reviewed in Sect. 3, provides the necessary playground for a precise description of linear transport in a two terminal device. The extention to transistor like configurations goes straightforwardly. On the other hand, the treatment of the non-equilibrium physics deserves special care, e.g. by using non-equilibrium Green functions and self-consistency arguments [46], which goes far beyond the limits of this short review.

Two main approximations have been tacitly assumed throughout this work: we have effectively employed (1) a single electron picture (2) in the limit of coherent transport. In doing so, we have been motivated by the fact that small molecules are typically well adsorbed to the leads providing a strong coupling. Here lies also the main difference with the 'artificial molecules' (obtained by confining the twodimensional electron gas at the interface of semiconducting heterostructures). In such mesoscopic devices, the electron states 
are strongly localized, thus the addition of any individual electron to the conducting island gives rise to the typical Coulomb staircase in the device $I-V$ characteristics [20]. Finally, the $\mathrm{C}_{60}$ calculations (Par. 4.3) have shown that the molecule clamped between the two mesoscopic leads undergoes to strong structural modification. Neglecting this effect, i.e., without properly relaxing the structure, leads to a substantial misestimation of the linear conductance. The modification of the flexible molecular structure under the effect of large bias voltages, and a direct computation of charge transfer problems are other important issues which were left out from the present work (for a recent account in this direction see [8]).

We can then conclude that different levels of investigation require different theoretical sophistication. A (semi)empirical description can be extremely useful in getting the flavor of the most qualitative effects, but can also be misleading. DFT codes may help in device parameter free calculations and have closer relevance to experiments. Besides that, it is evident that contacts can change dramatically the conductance profiles, and further progress in modelling is needed. It might indeed help to separate contact effects from 'molecular' effects. As far as leads are concerned, we have shown that low dimensional leads such as CNT do probe the conductance. Having a reliable model for the lead self-energy might be the key for cleaning (de-convoluting) spurious measurements.

Finally, we think that the addition of the richer physical environment that large molecules do experience might be easily scalable on top of the presented transport calculation scheme. This might be the case for molecular vibrations, and time dependent effects [47. More difficult would be the extension to comprise electron-electron interactions 48,49, and non-equilibrium relaxation. Attempts to cope with this latter challenge have to include a self-consistent or combined treatment of electronic transport and structural optimization [8].

\section{Acknowledgments}

B. C. Möbius is gratefully acknowledged for fruitful discussions. We are indebted to M. Albrecht for providing us with Fig. 2. GC research at MPI is sponsored by the Schloeßmann Foundation. FG and RG gratefully acknowledge financial support by the DFG under FOR 335.

\section{References}

1. M. A. Reed, Proc. IEEE 87, 652 (1999).

2. C. Joachim, J. K. Gimzewski, and A. Aviram, Nature 408, 541 (2000).

3. G. Cuniberti, G. Fagas, and K. Richter, Acta Phys. Pol. 32, 437 (2001).

4. K. W. Hipps, Science 294, 536 (2001). 
5. J. M. Seminario, C. E. De la Cruz, and P. A. Derosa, J. Am. Chem. Soc. 123, 5616 (2001).

6. G. Cuniberti, E. De Micheli, and G. Viano, Commun. Math. Phys. 16, 59 (2001); P. A. Derosa and J. M. Seminario, J. Phys. Chem. B 105, 471 (2001).

7. G. Cuniberti et al., Physica E 12, 749 (2002); R. Gutiérrez et al., Phys. Rev. B 65, 113410 (2002).

8. M. Di Ventra, S. T. Pantelides, and N. D. Lang, Phys. Rev. Lett. 88, 046801 (2002).

9. B. Kramer, Phys. Bl. 50, 543 (1994).

10. R. Landauer, IBM J. Res. Develop. 1, 223 (1957), reprinted in J. Math. Phys. 37, 5259 (1996).

11. M. Büttiker, IBM J. Res. Develop. 32, 317 (1988).

12. B. K. Nikolic, Phys. Rev. B 64, 165303 (2001).

13. S. Datta, Electronic Transport in Mesoscopic Systems (Cambridge University Press, Cambridge, 1999).

14. B. J. van Wees et al., Phys. Rev. Lett. 60, 848 (1988).

15. D. A. Wharam et al., J. Phys. C 21, L209 (1988).

16. Y. Meir and N. S. Wingreen, Phys. Rev. Lett. 68, 2512 (1992).

17. D. S. Fisher and P. A. Lee, Phys. Rev. B 23, R6851 (1981); T. N. Todorov, G. A. D. Briggs, and A. P. Sutton, J. Phys.-Condens. Matter 5, 2389 (1993).

18. A. D. Stone and A. Szafer, IBM J. Res. Develop. 32, 384 (1988).

19. S. Priyadarshi, S. S. Skourtis, S. M. Risser, and D. N. Beratan, J. Chem. Phys. 104, 9473 (1996).

20. Single Charge Tunneling, edited by H. Grabert and M. H. Devoret (Plenum Press, New York, 1992).

21. V. Mujica, M. Kemp, and M. A. Ratner, J. Chem. Phys. 101, 6856 (1994).

22. C. M. Goringe, D. R. Bowler, and E. Hernández, Rep. Prog. Phys. 60, 1447 (1997); S. Datta et al., Phys. Rev. Lett. 79, 2530 (1997); M. Magoga and C. Joachim, Phys. Rev. B 56, 4722 (1997); E. G. Emberly and G. Kirczenow, Phys. Rev. B 58, 10911 (1998); M. Paulsson and S. Stafström, J. Phys.-Condens. Matter 11, 3555 (1999); L. Chico, L. X. Benedict, S. G. Louie, and M. L. Cohen, Phys. Rev. B 54, 2600 (1996).

23. N. D. Lang and P. Avouris, Phys. Rev. Lett. 84, 358 (2000); S. N. Yaliraki et al., J. Chem. Phys. 111, 6997 (1999); J. J. Palacios, A. J. Perez-Jimenez, E. Louis, and J. A. Verges, Phys. Rev. B 64, 115411 (2001); H.-S. Sim, H.-W. Lee, and K. J. Chang, Phys. Rev. Lett. 87, 096803 (2001); J. Taylor, H. Guo, and J. Wang, Phys. Rev. B 63, 245407 (2001); P. S. Damle, A. W. Ghosh, and S. Datta, Phys. Rev. B 64, 201403 (2001).

24. R. Gutiérrez, F. Großmann, and R. Schmidt, Acta Phys. Pol. 32, 443 (2001).

25. R. Gutiérrez, F. Großmann, O. Knospe, and R. Schmidt, Phys. Rev. A 64, 013202 (2001).

26. M. Toerker et al., Submitted to Phys. Rev. B (2002).

27. N. D. Lang and P. Avouris, Phys. Rev. Lett. 81, 3515 (1998).

28. D. M. Newns, Phys. Rev. 178, 1123 (1969).

29. A. Karlsson et al., Nature 409, 150 (2001).

30. T. Rueckes et al., Science 289, 94 (2000); N. Yoneya, E. Watanabe, K. Tsukagoshi, and Y. Aoyagi, Appl. Phys. Lett. 79, 1465 (2001).

31. R. Saito, G. Dresselhaus, and M. S. Dresselhaus, Physical Properties of Carbon Nanotubes (World Scientific Publishing Co. Pte. Ltd., London, 1998). 
32. P. McEuen, Phys. World 13, 31 (2000).

33. V. Derycke, R. Martel, J. Appenzeller, and P. Avouris, Nano Letters 1, 453 (2001); H. W. C. Postma et al., Science 293, 76 (2001); R. Martel et al., Appl. Phys. Lett. 73, 2447 (1998).

34. H. Watanabe, C. Manabe, T. Shigematsu, and M. Shimizu, Appl. Phys. Lett. 78, 2928 (2001). S. S. Wong et al., Nature 394, 52 (1998).

35. H. Nishijima et al., Appl. Phys. Lett. 74, 4061 (2000).

36. S. Akita, H. Nishijima, T. Kishida, and Y. Nakayama, Jpn. J. Appl. Phys. 39, 7086 (2000); A. I. Onipko et al., Phys. Rev. B 61, 11118 (2000); A. L. Vázquez de Parga et al., Phys. Rev. Lett. 80, 357 (1998).

37. C. Thelander et al., Appl. Phys. Lett. 79, 2106 (2001); M. P. Anantram, S. Datta, and Y. Xue, Phys. Rev. B 61, 14219 (2000); P. J. de Pablo et al., Appl. Phys. Lett. 74, 323 (1999).

38. G. Cuniberti, G. Fagas, and K. Richter, Chem. Phys. 281, 465 (2002).

39. G. Cuniberti, J. Yi, and M. Porto, Appl. Phys. Lett. 81, 850 (2002); J. Yi, G. Cuniberti, and M. Porto, Submitted (2002).

40. V. Mujica, M. Kemp, and M. A. Ratner, J. Chem. Phys. 101, 6849 (1994).

41. A. Nitzan, Ann. Rev. Phys. Chem. 52, 681 (2001); L. E. Hall, J. R. Reimers, N. S. Hush, and K. Silverbrook, J. Chem. Phys. 112, 1510 (2000).

42. R. Saito, M. Fujita, G. Dresselhaus, and M. S. Dresselhaus, Phys. Rev. B 46, 1804 (1992); P. R. Wallace, Phys. Rev. 71, 622 (1947).

43. M. S. Ferreira, T. G. Dargam, R. B. Muniz, and A. Latgé, Phys. Rev. B 63, 245111 (2001).

44. H. J. Choi and J. Ihm, Solid State Commun. 111, 385 (1999).

45. C. Zeng, H. Wang, B. Wang, and J. G. Hou, Appl. Phys. Lett. 77, 3595 (2000).

46. Y. Xue, S. Datta, and M. A. Ratner, To appear in Chem. Phys. (2002).

47. G. Cuniberti, A. Fechner, M. Sassetti, and B. Kramer, Europhys. Lett. 48, 66 (1999).

48. H. J. Schulz, G. Cuniberti, and P. Pieri, in Field theories for low-dimensional condensed matter systems, edited by G. Morandi et al. (Springer, Berlin, 2000).

49. G. Cuniberti, M. Sassetti, and B. Kramer, Europhys. Lett. 37, 421 (1997). 